\title{
ANÁLISE PERCEPTIVO-AUDITIVA DE PARÂMETROS VOCAIS EM CANTORES DA NOITE DO ESTILO MUSICAL BREGA DA CIDADE DO RECIFE
}

\author{
Perceptual vocal pattern analysis of singers from \\ kitschy musical style in Recife
}

Elthon Gomes Fernandes da Silva ${ }^{(1)}$, Carmem Lúcia Cerqueira de Luna ${ }^{(2)}$

\begin{abstract}
RESUMO
Objetivo: avaliar de forma perceptivo-auditiva a voz dos cantores da noite do estilo musical Brega da cidade do Recife. Métodos: pesquisa realizada na clínica-escola do curso de Fonoaudiologia da Universidade Federal de Pernambuco e na emissora de TV Rede Estação canal 14, ambos localizados na cidade do Recife. Trata-se de estudo observacional, transversal e descritivo. Com anuência de 13 cantores, maiores de 18 anos, houve gravação da voz falada na emissão sustentada de vogais e durante a música "parabéns pra você"; na voz cantada realizou-se a gravação de trecho de música pertencente ao repertório do cantor. Resultados: tempos de fonação reduzidos; modificações no pitch e loudness, comparando voz falada e cantada, ambos passando de adequados para, respectivamente, agudo e elevada; mudanças na ressonância, que era laringofaríngea e tornou-se equilibrada com compensação nasal. Houve manutenção do ataque vocal brusco; mudança do registro modal misto na voz habitual para o modal cabeça na voz profissional; predominância da qualidade vocal clara na voz falada e padrões adequados para modulação, projeção e articulação na voz cantada. Conclusão: os cantores da noite do estilo musical Brega da cidade do Recife apresentaram tempos de fonação reduzidos e tiveram, da voz falada para a voz cantada, mudanças no pitch, loudness e ressonância e manutenção das características vocais para ataque e registro. A qualidade vocal clara na voz falada foi predominante, assim como a modulação adequada, boa projeção e articulação precisa estavam entre os padrões vocais mais frequentes na voz cantada.
\end{abstract}

DESCRITORES: Voz; Avaliação; Música

\section{INTRODUÇÃO}

A análise perceptivo-auditiva da voz representa uma ferramenta básica para caracterizar um tipo de voz ${ }^{1}$, sendo uma forma que permite o fonoaudiólogo ajudar o paciente em sua solicitação e estabelecer uma proposta terapêutica adequada ${ }^{2}$. A partir desta premissa, a avaliação se configura como o marco inicial para o diagnóstico e tratamento de

(1) Fonoaudiólogo; Mestrando no Programa de Pós-Graduação em Saúde Humana e Meio Ambiente na Universidade Federal de Pernambuco, UFPE, Recife, PE.

(2) Fonoaudióloga do Hospital Getúlio Vargas e do Hospital dos Servidores do Estado, Recife, PE; Docente do Curso de Fonoaudiologia da Fundação de Ensino Superior de Olinda, FUNESO, Olinda, PE; Especialista em voz pela universidade Federal de Pernambuco.

Conflito de interesse: inexistente uma alteração ou patologia da voz bem como para o seu aperfeiçoamento, mantendo sua importância durante o processo de evolução do indivíduo para assegurar o momento adequado de finalização da terapia ${ }^{3}$.

Com o recurso deste tipo de avaliação, numa posterior escuta detalhada, é possível detectar as particularidades da produção vocal (na fala ou no canto) que poderiam passar despercebidas pelo terapeuta se este não tivesse registrado, seja por meio de fitas ou por registro digital, o momento que o paciente realizou a emissão. Ressalta-se que para a voz cantada, além deste modo de avaliação, foi recentemente mostrada a importância da fonetografia ${ }^{4}$ como instrumento auxiliador para uma classificação vocal adequada.

A produção da voz falada e da voz cantada necessita de estruturas anatômicas semelhan- 
tes, porém estas sofrem adaptações em seu funcionamento ${ }^{5}$. Por meio de comparação entre voz falada e cantada, percebe-se algumas das diferenças existentes entre estes dois tipos de voz quando analisam-se: os tipos de ciclos inspiratórios e expiratórios necessários (sendo mais rápidos na voz cantada); a fonação, que produz quantidade regular de harmônicos na voz falada; as pausas e velocidade, que dependem de treinamento específico na voz cantada e também a projeção de voz, em que a emissão na voz cantada pode variar de 45 a $110 \mathrm{~dB}$ de intensidade quando o cantor precisa ir de um pianíssimo ao fortíssimo ${ }^{6}$.

As características vocais encontradas na avaliação perceptivo-auditiva em profissionais da voz falada ou cantada podem ser decorrentes de padrão vocal necessário para manter-se em sua ocupação ou até mesmo para funcionar como um "sinal de identidade" em seu meio profissional. Ressalta-se que o uso da voz visando reconhecimento em seu ambiente profissional pode ter relação com o sentimento de importância em executar a sua profissão e também com a psicodinâmica vocal do indivíduo. A literatura mostra que a partir do significado que é atribuído às suas relações sociais, o sujeito pode criar uma voz peculiar e que desempenhe uma função específica perante as experiências que estabelece em sua vida ${ }^{7}$ do mesmo modo que durante um processo terapêutico, no momento de escuta do novo tipo voz, o sujeito pode experimentar diferentes maneiras de uso, criando novas formas de ser reconhecido no mundo ${ }^{8}$. A busca dos cantores pelo sucesso pode estar relacionada a aspectos como desejo de melhor condição financeira, reconhecimento do seu talento, necessidade de ser conhecido nos vários ambientes que frequenta no dia-a-dia e de estar constantemente na mídia. Tais aspectos impulsionam atitudes que seguem desde a escolha de uma música visando a aceitação de um público-alvo, até a preferência de uma performance no canto que favoreça o seu destaque, quando comparado a outros cantores de mesmo estilo musical, mesmo que ocorram sintomas prejudiciais à voz depois das apresentações.

Uma questão interessante no ritmo Brega é o fato de haver uma "democratização" da condição de artista, ou seja, praticamente qualquer pessoa pode ser um astro do Brega uma vez que para tal pretensão não é exigido domínio de técnicas especiais para a produção artística: compositores não precisam saber escrever e cantores não precisam necessariamente saber cantar ${ }^{9}$. E isto se torna um ponto importante de investigação do fonoaudiólogo ao atender um cantor deste estilo, pois é comum encontrar, entre os profissionais da voz, aqueles que nunca procuraram um auxílio específico e no caso dos cantores, nunca terem feito aulas de canto ou mesmo uma terapia fonoaudiológica. Outros profissionais da voz como operadores de telemarketing ${ }^{10}$ e professores ${ }^{11}$ também são citados como grupos que pouco conhecem sobre o uso profissional da voz.

A falta de um acompanhamento específico para o canto pode acarretar padrões inadequados que podem interferir no desempenho durante os shows ocasionando a disodia, definida como alterações que ocorrem na prática do canto ${ }^{12}$, e até, nas situações mais graves, episódios de rouquidão de grau moderado pós-apresentação. Esta deve ser uma informação relevante no momento de uma anamnese com cantor, pois já foi descrita na literatura que nódulos e edema de Reinke, patologias resultantes de mau uso e abuso da voz associados à falta de cuidados básicos para manter a saúde vocal, estão entre os principais diagnósticos quando se realizam exames de imagem da laringe neste grupo de profissionais ${ }^{13,14}$ assim como já foi apresentado que há concordância entre os achados de uma avaliação fonoaudiológica perceptivo-auditiva com as avaliações médicas ${ }^{15}$.

O objetivo desta pesquisa foi avaliar de forma perceptivo-auditiva a voz dos cantores da noite do estilo musical Brega da cidade do Recife.

\section{MÉTODOS}

Trata-se de um estudo observacional, transversal e descritivo, com desenho do tipo série de casos.

Entre os meses de junho e agosto de 2007 foram avaliados 13 cantores da noite do estilo musical Brega da cidade do Recife, de ambos os sexos. Os critérios de inclusão foram: ser maior de 18 anos e cantar neste estilo musical. Foram excluídos aqueles cantores que tinham idade menor que 18 anos e não pertenciam ao estilo musical estudado.

O presente estudo foi realizado na clínica-escola do curso de Fonoaudiologia da Universidade Federal de Pernambuco e na emissora de TV Rede Estação canal 14, ambos localizados na cidade do Recife.

Com o objetivo de avaliar parâmetros vocais relacionados à voz falada: pitch, loudness, ataque vocal, ressonância, registro, qualidade vocal e tempo máximo de fonação, foi realizada a gravação da emissão sustentada das vogais /a/, /i/ e /u/ e da música "parabéns pra você". Para a avaliação da voz cantada realizou-se a gravação, à capela, de um trecho de música pertencente ao seu repertório para avaliação dos seguintes parâmetros: pitch, loudness, ataque vocal, ressonância, registro, modulação, projeção e articulação. O resultado das avaliações de cada voluntário era registrado em protocolo (Figura 1). 


\section{PROTOCOLO DE AVALIAÇÃO PERCEPTIVO-AUDITIVA VOZ FALADA E VOZ CANTADA}

Nome

Idade

Data / /

(VOZ FALADA)

1) Pitch:

$\square$ Agudo $\square$ Adequado $\square$ Grave

2) Loudness:

$\square$ Reduzida $\square$ Adequada $\square$ Elevada

3) Ataque vocal:

$\square$ Suave $\square$ Brusco $\square$ Aspirado

4) Ressonância:

$\square$ Equilibrada $\square$ Laringo-faríngea $\square$ Excessivamente oral

$\square$ Excessivamente nasal

5) Registro: $\quad \square$ Basal $\square$ Modal do tipo peito $\square$ Modal do tipo misto

$\square$ Modal do tipo cabeça $\square$ Elevado

6) Qualidade vocal: $\square$ Rouca $\square$ Clara $\square$ Nasal

7) Tempo Máximo de Fonação (TMF)
la/
/i/
segundos
/u/ _ segundos

\section{(VOZ CANTADA)}

1) Pitch:

$\square$ Agudo $\square$ Adequado $\square$ Grave

2) Loudness:

$\square$ Reduzida $\square$ Adequada $\square$ Elevada

3) Ataque vocal:

$\square$ Suave $\square$ Brusco $\square$ Aspirado

4) Ressonância: $\square$ Equilibrada $\square$ Laringo-faríngea $\square$ Excessivamente oral

$\square$ Excessivamente nasal

5) Registro: $\quad \square$ Basal $\square$ Modal do tipo peito $\square$ Modal do tipo misto

$\square$ Modal do tipo cabeça $\square$ Elevado

6) Modulação: $\quad \square$ Voz com modulação adequada $\quad \square$ Voz com modulação inadequada

7) Projeção: $\quad \square$ Voz bem projetada $\quad \square$ Voz mal projetada $\quad \square$ Voz sem projeção

8) Articulação: $\quad \square$ Precisa $\square$ Imprecisa

Figura 1 - Protocolo de avaliação vocal

A pesquisa foi aprovada pelo Comitê de Ética em Pesquisa com seres Humanos do Centro de Ciências da Saúde da Universidade Federal de Pernambuco (CEP/CCS/UFPE) sob número de processo $n^{\circ}$ 126/2007.

Foi utilizado o programa Epilnfo versão 3.4.3 (nov, 2007) para o banco de dados e posterior análise das informações coletadas que foram submetidas à análise estatística descritiva e serão apresentadas por meio da distribuição em frequência absoluta e frequência relativa, para os resultados sobre os parâmetros vocais, e em valores de mínimo, máximo e média, para os tempos de fonação.

\section{RESULTADOS}

A população de estudo constou de seis homens (46\%) e sete mulheres (54\%), com média de idade de 25,5 anos para o sexo masculino e 22,71 anos para o feminino. Ambos os grupos apresentaram tempos máximo de fonação reduzidos. O tempo máximo de fonação (TMF) médio, em segundos (seg), nas vogais /a/, /i/ e /u/ foi respectivamente de 6,96 seg; 6,05 seg e 5,84 seg para os homens e 7,57 seg; 6,12 seg e 6,16 seg para as mulheres (Tabela 1).

Foi observado que na voz falada 11 cantores tiveram predominância de pitch adequado ao sexo 
e à idade (84,6\%), 10 evidenciaram loudness adequada $(76,9 \%)$ e 12 apresentaram ataque vocal brusco $(92,3 \%)$. Já na voz cantada a preferência do ajuste vocal de oito cantores estava no pitch agudo $(61,5 \%)$, a mesma quantidade utilizou loudness elevada $(61,5 \%)$ e 12 participantes apresentaram ataque vocal brusco (92,3\%) (Tabela 2). Embora este último achado, nos dois tipos de voz avaliados, possa originar a hipótese de prejuízo na qualidade vocal, somente foi observada rouquidão em um dos cantores $(7,7 \%)$, ocorrendo na voz falada o predomínio da qualidade vocal clara em 12 cantores $(92,3 \%)$ (Tabela 3).

O parâmetro de registro vocal modal do tipo misto foi o mais utilizado por 11 cantores na voz habitual $(84,6 \%)$, entretanto na voz profissional o registro modal do tipo cabeça foi predominante em oito participantes $(61,5 \%)$ (Tabela 2$)$.

Em análise da ressonância na voz falada, foi visto que todos os cantores (100\%) apresentaram ressonância laringo-faríngea e destes, apenas dois $(15,4 \%)$ demonstraram uso de foco nasal em momentos isolados durante a emissão das vogais /i/ e /u/. Esta situação difere ao ser avaliada a voz cantada: sete cantores $(53,8 \%)$ apresentaram ressonância equilibrada com uso de foco nasal quando necessitaram atingir os agudos em determinados momentos da música, e três participantes $(23,1 \%)$ usaram a ressonância laringo-faríngea com compensação nasal para emitirem os agudos (Tabela 2).

A maioria dos cantores demonstrou, na voz cantada, resposta adequada no que se refere à modulação, projeção e articulação, apresentando, respectivamente, 12 com modulação adequada $(92,3 \%), 10$ utilizando a voz bem projetada $(76,9 \%)$ e 13 com articulação precisa (100\%) (Tabela 4).

\section{DISCUSSÃO}

O TMF é um indício de como a laringe se comporta frente à força aerodinâmica exercida durante a passagem do ar vindo dos pulmões, sendo o tempo de emissão vocal diretamente proporcional à capacidade de controle na saída do fluxo de ar expirado. De acordo com a literatura ${ }^{16}$, valores esperados para homens e mulheres adultos são de 20 a 35 segundos e 14 a 25 segundos, respectivamente. Entretanto, os participantes deste estudo apresentaram valores abaixo do esperado para idade e sexo.

Deve-se ressaltar que o canto exige uma adequada coordenação entre o sistema respiratório e a fonação ${ }^{17}$, em que a habilidade na regulagem da expiração favorece ao cantor a competência para sustentar uma nota ${ }^{18}$. É durante a sustentação de uma nota musical que ficam mais perceptíveis muitas das características vocais e também as deficiências na realização das técnicas que o cantor utiliza, o que pode gerar admiração ou crítica do espectador a respeito da sua atuação. Não sustentar de modo preciso uma nota musical no fim de uma estrofe reflete uma sensação de fadiga e pouco controle na voz, o que pode comprometer a intenção comunicativa existente na música e o surgimento das emoções que esta causaria no ouvinte.

Os achados sobre a mudança do padrão do pitch e loudness dos cantores, entre a voz falada e a voz cantada, podem ser o início do conhecimento das características vocais que ocorrem durante 0 canto numa música do ritmo brega.

Enquanto apenas um participante $(7,7 \%)$ utilizava o pitch agudo na voz falada, a preferência por este parâmetro na voz cantada aumentava para oito $(61,5 \%)$. E o número reduzido de três $(23,1 \%)$ cantores que utilizavam loudness elevada durante a voz falada, foi modificado para oito $(61,5 \%)$ na voz cantada.

Para que estas alterações aconteçam são necessárias variações na dinâmica da produção da voz. A literatura respalda esta hipótese quando apresenta que a fisiologia na mudança do pitch envolve uma complexa inter-relação entre estruturas respiratórias, laríngeas e supralaríngeas ${ }^{19}$. A emissão de sons agudos está relacionada à posição mais elevada da laringe, com consequente diminuição do trato vocal ${ }^{16,20}$, e associada ao aumento da rigidez das pregas vocais, especifica-

Tabela 1 - Distribuição do tempo máximo de fonação, em segundos, encontrado nos cantores do sexo masculino $(n=06)$ e feminino $(n=07)$ do estilo musical Brega da cidade do Recife

\begin{tabular}{ccccccc}
\hline \multirow{2}{*}{ VOGAL } & \multicolumn{3}{c}{ MASCULINO } & \multicolumn{3}{c}{ FEMININO } \\
\cline { 2 - 7 } & Mínimo & Máximo & Média & Mínimo & Máximo & Média \\
\hline /a/ & 3,7 & 8,86 & 6,96 & 5,42 & 9,45 & 7,57 \\
/i/ & 4,35 & 7,30 & 6,05 & 4,47 & 7,70 & 6,12 \\
/u/ & 4,12 & 8,56 & 5,84 & 5,07 & 7,99 & 6,16 \\
\hline
\end{tabular}


Tabela 2 - Distribuição do pitch, loudness, ataque vocal, registro e ressonância da voz falada e cantada dos cantores da noite do estilo musical Brega da cidade do Recife

\begin{tabular}{|c|c|c|c|c|}
\hline \multirow{2}{*}{ PARÂMETROS } & \multicolumn{2}{|c|}{ VOZ FALADA } & \multicolumn{2}{|c|}{ VOZ CANTADA } \\
\hline & $F(N)$ & $F(\%)$ & $F(N)$ & $F(\%)$ \\
\hline \multicolumn{5}{|l|}{ PITCH } \\
\hline Agudo & 1 & 7,7 & 8 & 61,5 \\
\hline Adequado ao sexo e à idade & 11 & 84,6 & 4 & 30,8 \\
\hline Grave & 1 & 7,7 & 1 & 7,7 \\
\hline \multicolumn{5}{|l|}{ LOUDNESS } \\
\hline Reduzida & - & - & 1 & 7,7 \\
\hline Adequado & 10 & 76,9 & 4 & 30,8 \\
\hline Elevada & 3 & 23,1 & 8 & 61,5 \\
\hline \multicolumn{5}{|l|}{ ATAQUE VOCAL } \\
\hline Suave & - & - & 1 & 7,7 \\
\hline Brusco & 12 & 92,3 & 12 & 92,3 \\
\hline Aspirado & 1 & 7,7 & - & - \\
\hline \multicolumn{5}{|l|}{ REGISTRO } \\
\hline Basal & - & - & - & - \\
\hline Modal do tipo peito & 1 & 7,7 & 1 & 7,7 \\
\hline Modal do tipo misto & 11 & 84,6 & 4 & 30,8 \\
\hline Modal do tipo cabeça & 1 & 7,7 & 8 & 61,5 \\
\hline Elevado & - & - & - & - \\
\hline \multicolumn{5}{|l|}{ RESSONÂNCIA } \\
\hline Equilibrada & - & - & - & - \\
\hline $\begin{array}{l}\text { Equilibrada com } \\
\text { compensação nasal }\end{array}$ & - & - & 7 & 53,8 \\
\hline Laringo-faríngea & 11 & 84,6 & 2 & 15,4 \\
\hline $\begin{array}{l}\text { Laringo-faríngea com } \\
\text { compensação nasal }\end{array}$ & 2 & 15,4 & 3 & 23,1 \\
\hline Faríngea & - & - & - & - \\
\hline Excessivamente oral & - & - & - & - \\
\hline Excessivamente nasal & - & - & 1 & 7,7 \\
\hline
\end{tabular}

Tabela 3 - Caracterização da qualidade vocal da voz falada dos cantores da noite do estilo musical Brega da cidade do Recife

\begin{tabular}{lcc}
\hline PARÂMETRO & $\mathbf{F}(\mathbf{N})$ & $\mathbf{F}(\%)$ \\
\hline QUALIDADE VOCAL & & \\
Rouca & 1 & 7,7 \\
Clara & 12 & 92,3 \\
Nasal & - & - \\
\hline
\end{tabular}

Tabela 4 - Padrões de modulação, projeção e articulação encontrados na voz cantada em cantores da noite do estilo musical Brega da cidade do Recife

\begin{tabular}{lcc}
\hline PARÂMETROS & $\mathbf{F ( N )}$ & $\mathbf{F}(\%)$ \\
\hline $\begin{array}{l}\text { MODULAÇÃO } \\
\text { Voz com modulação }\end{array}$ & 12 & 92,3 \\
$\quad \begin{array}{l}\text { adequada } \\
\text { Voz com modulação }\end{array}$ & 1 & 7,7 \\
$\quad$ inadequada & & \\
PROJEÇÃO & & \\
$\quad$ Voz bem projetada & 10 & 76,9 \\
$\quad$ Voz mal projetada & 3 & 23,1 \\
$\quad$ Voz sem projeção & - & - \\
ARTICULAÇÃO & & \\
$\quad$ Precisa & 13 & 100 \\
Imprecisa & - & - \\
\hline
\end{tabular}


mente dentro dos músculos tireoaritenóideos ${ }^{21}$. Já a loudness elevada, modelo preferido pelos cantores desta pesquisa na voz cantada, tem associação com a pressão subglótica exercida pela corrente de ar expirado ${ }^{20 .}$

Diante do que foi apresentado anteriormente, é oportuno expor que cantar numa loudness elevada exige uma boa capacidade respiratória e tal capacidade não foi apresentada pelos cantores. Assim, é exigida maior atividade da fonte glótica, adução intensa, porque o abastecimento de ar não foi suficiente para aumentar a pressão subglótica e facilitar o aumento da intensidade vocal, com isso é sugestiva a hipótese de que esta situação favoreceu o ataque vocal brusco em 12 cantores (92,3\%) na voz cantada.

Uma alternativa para a elevação da loudness na voz cantada sem prejuízos à laringe, é a realização de aquecimento vocal. Foi descrito na literatura ${ }^{22}$ que após submeter cantores de heavy metal, que atuavam na cidade do Recife, a um programa de aquecimento vocal, a loudness ficou elevada, levando os autores a inferirem que tal êxito se deve ao fato de que os exercícios para aquecimento podem ter aumentado a pressão subglótica e, conse quentemente, elevado a loudness.

Durante avaliação perceptivo-auditiva, a qualidade vocal clara foi evidenciado por 12 cantores $(92,3 \%)$. Sabe-se que os resultados sobre o parâmetro qualidade vocal são influenciados pelas condições que o trato vocal se comporta durante a avaliação ${ }^{23}$. Além disso, este também é capaz de realizar, diante de um quadro de disfonia funcional ou organofuncional, ajustes compensatórios que comprometam uma avaliação ou uma reabilitação ${ }^{24}$. Diante destas informações, a prevalência da qualidade vocal clara na voz falada dos cantores pesquisados sugere reduzidas chances de serem encontradas alterações orgânicas que acometeram as estruturas que compõem o aparelho fonador.

Sendo cada registro composto de uma faixa de frequência quase uniforme, numa avaliação, é possível fazer a distinção entre um registro e outro ${ }^{25}$. A preferência de oito cantores $(61,5 \%)$ pelo registro modal do tipo cabeça, na qual a posição alta da laringe facilita a produção de sons agudos ${ }^{23}$, é mais um indicativo da necessidade do cantor do ritmo Brega em adquirir este padrão de sons vocais.

De acordo com o estilo, o trato vocal pode assumir diferentes configurações, uma vez que o cantor precisa valorizar determinados harmônicos durante as músicas do seu repertório.

Ocorre a possibilidade de na avaliação do foco ressonantal, serem encontrados focos duplos ${ }^{25}$. $\mathrm{Na}$ voz falada, a presença de foco nasal em 2 cantores, especificamente nas vogais /i/ e /u/, podem ter relação com a frequência sonora e a posição de órgãos fonoarticulatórios. Ambas já foram descritas na literatura com formantes faríngeos em torno de $300 \mathrm{~Hz}$ e embora a vogal /i/ seja mais aguda e tenha maior ressonância oral em relação à vogal $/ \mathrm{u} /{ }^{26}$, as duas são classificadas como vogais altas, pois a língua está em posição mais alta no trato vocal diminuindo o espaço para a saída do ar pela cavidade oral ${ }^{27}$. Com isso, possivelmente houve o direcionamento do ar para a cavidade nasal num determinado momento da emissão destas vogais, resultando na observação de dois focos de ressonância e sugerindo um ajuste inadequado para a boa produção vocal da voz falada.

Condição semelhante foi encontrada na voz falada de pessoas com alterações no funcionamento da tireóide, em que a ressonância laringofaríngea com foco nasal compensatório teve a seguinte justificativa: devido ao maior esforço e tensão durante a fonação, o foco nasal surge na tentativa de amenizar essa tensão e elevar a qualidade da emissão ${ }^{28}$.

A configuração da cavidade nasal, por ser menor que a cavidade oral, favorece os sons de frequência elevada ${ }^{5} \mathrm{e}$ os seios paranasais atuam de forma secundária para o espectro nasal ${ }^{29}$. Embora seja uma região facilitadora para a impressão acústica de sons agudos, estudo realizado com a vogal /a/, numa emissão vocal direcionada para a cavidade nasal, revelou perda da intensidade, em decibéis, no primeiro formante e realce do espectro nos formantes ao redor de $3 \mathrm{KHz}{ }^{30}$.

A abertura de boca durante a emissão dos agudos é uma alternativa para que não haja uma nasalidade excessiva ${ }^{5}$. Tal recurso poderia ser utilizado pelos cantores desta pesquisa que apresentaram a ressonância laringo-faríngea e equilibrada, ambas com compensação nasal, no momento necessário para atingir as notas mais altas. Entretanto, esta característica se mostra como indicador vocal de um(a) cantor(a) do estilo brega revelando que este pode ser um padrão vocal da sua profissão e provavelmente numa terapia fonoaudiológica o intuito seria de favorecer a melhor produção de acordo com as exigências do seu ritmo, sendo a ressonância equilibrada com compensação nasal uma condição ideal para o cantor.

Sabe-se que as condições psicológicas do cantor interferem no controle da modulação, pois os momentos de grande agitação podem ser acompanhados de mudanças imprevisíveis na altura da voz, da mesma forma que excesso de tranquilidade ocasiona prejuízo às mudanças bruscas de frequência ${ }^{31}$. 
Na voz falada do locutor de rádio, este parâmetro permite o ouvinte manter efetivamente o interesse no texto que é dito ${ }^{32}$. Já na voz cantada, a modulação vocal adequada, presente na maioria dos cantores, indica habilidade de variar os tons promovendo a inflexão necessária para transmitir a mensagem da música e obedecer à melodia definida pelos instrumentos musicais.

A voz bem projetada e articulação precisa, características encontradas em, respectivamente, $10(76,9 \%)$ e $13(100 \%)$ cantores de brega desta pesquisa, são recursos positivos para a voz cantada. Ter uma articulação precisa indica controle do processo fonoarticulatório ${ }^{33}$, torna-se essencial para compreensão da mensagem pelo ouvinte e, associada à boa projeção vocal, nos casos de uso da voz em grandes ambientes, fará com que a voz se dissipe mais amplamente no espaço em questão ${ }^{34}$.

\section{CONCLUSÃO}

Os cantores da noite do estilo musical Brega da cidade do Recife apresentaram tempos de fonação reduzidos e tiveram, da voz falada para a voz cantada, mudanças no pitch, loudness e ressonância e manutenção das características vocais para ataque e registro. A qualidade vocal clara na voz falada foi predominante, assim como a modulação adequada, boa projeção e articulação precisa estavam entre os padrões vocais mais fre quentes na voz cantada.

\section{AGRADECIMENTOS}

Nosso agradecimento à equipe do programa Tarde Legal e à emissora de TV Rede Estação canal 14, pela contribuição que se tornou o ponto fundamental para a realização desta pesquisa.

\begin{abstract}
Purpose: to evaluate the perceptual form concerning the voice of the singers from kitschy musical style in Recife. Methods: clinical research was carried out in the clinic-school of Speech, Language and Hearing Sciences course in the Federal University of Pernambuco and Network TV Station channel 14 , both located in the city of Recife. This is an observational, transversal and descriptive study. With consent of 13 singers, above 18-year old, there was recording of the spoken voice while speaking vowel as well during the song "happy birthday"; as for singing voice, there was recording through songs from singer's repertory. Results: times of speech reduced; pitch and loudness modifications, comparing spoken and singing voice, both were adequate but changed into sharp and high, respectively; changes in resonance, of laryngeal-pharyngeal type to balanced with nasal compensation. There was maintenance of the brusque vocal attack; change of the mixed modal register in habitual voice to head modal in professional voice; clear vocal quality predominance in spoken voice and patterns adequate to modulation, projection and articulation in singing. Conclusion: the singers from kitschy musical style in Recife had reduced maximum phonation time and had, in the speech voice to singing voice, changes in pitch, loudness and resonance and maintenance of vocal characteristics for attack and recording. A clear voice quality in speech voice was predominant and the appropriate modulation, good projection and good articulation were among the most frequent patterns in singing voice.
\end{abstract}

KEYWORDS: Voice; Evaluation; Music

\section{REFERÊNCIAS}

1. Andrada e Silva MA, Duprat AC. Voz cantada. In: Ferreira LP, Befi-Lopes DM, Limongi SCO, organizador. Tratado de fonoaudiologia. São Paulo: Roca; 2004. p. 177-94.

2. Souza DPD, Silva APBV, Jarrus ME, Pinho SMR. Avaliação fonoaudiológica vocal em cantores infanto-juvenis. Rev. CEFAC. 2006; 8(2):216-22.
3. Cielo CA, Cappellari VM. Tempo máximo de fonação de crianças pré-escolares. Rev Bras Otorrinolaringol. 2008; 74(4):552-60.

4. Camargo TF, Barbosa DA, Teles LCS. Características da fonetografia em coristas de diferentes classificações vocais. Rev Soc Bras Fonoaudiol. 2007; 12(1):10-7.

5. Lopes LW. Análise da ampliação da tessitura vocal em cantores. 2004. [monografia]. Recife (PE): Universidade Federal de Pernambuco; 2004. 
6. Behlau M, Rehder M I. Higiene vocal para o canto coral. Rio de Janeiro: Revinter; 1997. 44p.

7. Vilela FCA, Ferreira LP. Voz na clínica fonoaudiológica: grupo terapêutico como possibilidade. Dist Comun. 2006; 18(2):235-43.

8. Cunha MC, Pinheiro MG. Voz e psiquismo: diálogos entre fonoaudiologia e psicanálise. Dist Comun. 2004; 16(1):83-91.

9. Fontanella FI. A estética do brega: cultura de consumo e o corpo nas periferias do Recife [dissertação]. Recife (PE): Universidade Federal de Pernambuco; 2005.

10. Ferreira LP, Akutsu CM, Luciano $P$, Viviano NAG. Condições de produção vocal de teleoperadores: correlação entre questões de saúde, hábitos e sintomas vocais. Rev Soc Bras Fonoaudiol. 2008; 13(4):307-15.

11. Penteado RZ, Pereira IMTB. Qualidade de vida e saúde vocal de professores. Rev. Saúde Pública. 2007; 41(2):236-43.

12. Oliveira CM, Pinho SMR, Ferreira V. A relação entre música e fonoaudiologia, com ênfase no estilo gospel. J Bras Fonoaudiol. 2001; 3(9):271-7.

13. Fortes FSG, Imamura R, Tsuji DH, Sennes LU. Perfil dos profissionais da voz com queixas vocais atendidos em um centro terciário de saúde. Rev Bras Otorrinolaringol. 2007; 73(1):27-31.

14. Silverio KCA, Gonçalves CGO, Penteado RZ, Vieira TPG, Libardi A, Rossi D. Ações em saúde vocal: proposta de melhoria do perfil vocal de professores. Pró-Fono. 2008; 20(3):177-82.

15. Nemr K, Amar A, Abrahäo M, Leite GCA, Köhle JS, Santos AO, Correa LAC. Análise comparativa entre avaliação fonoaudiológica perceptivo-auditiva, análise acústica e laringoscopias indiretas para avaliação vocal em população com queixa vocal. Rev Bras Otorrinolaringol. 2005; 71(1):13-7.

16. Behlau M, Pontes P. Avaliação e tratamento das disfonias. São Paulo: Lovise; 1995. 290p.

17. Lundy DS, Roy S, Casiano RR, Evans J, Sullivan PA, Xue JW. Relationship between aerodynamic measures of glottal efficiency and stroboscopic findings in asymptomatic singing students. J Voice. 2000; 14(2):178-83.

RECEBIDO EM: 22/10/2008

ACEITO EM: 12/05/2009

Endereço para correspondência:

Elthon Gomes Fernandes da Silva

Rua Bela Vista, 108

Olinda - PE

CEP: 53120-040

E-mail: elthonfernandes@yahoo.com.br
18. Estienne F. Voz falada, voz cantada: avaliação e terapia. Rio de Janeiro: Revinter; 2004. 196p.

19. Lam Tang JA, Boliek CA, Rieger JM. Laryngeal and respiratory behavior during pitch change in professional singers. J Voice. 2008; 22(6):622-33.

20. Pinho SMR. Fundamentos em fonoaudiologia: tratando os distúrbios da voz. Rio de Janeiro: Guanabara-Koogan; 1998. 128p.

21. Jiang J, Lin E, Hanson DG. Vocal fold physiology. Otolaryngol Clin North Am. 2000; 33(4):699-718.

22. Lira AFS, Silva RO. Efeito dos exercícios de aquecimento e desaquecimento vocal em cantores de heavy metal [monografia]. Recife (PE): Faculdade Integrada do Recife; 2007.

23. Barros APB, Carrara-de-Angelis E. Avaliação perceptivo-auditiva da voz. In: Dedivitis RA, Barros APB. Métodos de avaliação e diagnóstico de laringe e voz. São Paulo: Lovise; 2002. p. 39-52.

24. Magri A, Cukier-Blaj S, Karman DF, Camargo ZA. Correlatos perceptivos e acústicos dos ajustes supraglóticos na disfonia. Rev. CEFAC. 2007; 9(4):512-8. dx.doi.org/ S1516-18462007000400012

25. Sant'Ana FL. Aspectos fisiológicos da produção vocal em falsete. Rev. CEFAC. 2000; 2(1):97-101.

26. Brandi E. O controle da voz na fala. In: Brandi E. Educação da voz falada: a terapêutica da conduta vocal. 4. ed. São Paulo: Atheneu; 2002. p. 79-100.

27. Mussalim F, Bentes AC, organizadores. Introdução à linguística: domínios e fronteiras. vol. 1. 5. ed. São Paulo: Cortez; 2005. 294 p.

28. Isolan-Cury RW, Silva MAM, Monte O, Cury AN. Caracterização vocal de pacientes com hipertireoidismo e hipotireoidismo. Rev Soc Bras Fonoaudiol. 2007; 12(2):135-40.

29. Dang J, Honda K, Suzuki H. Morphological and acoustical analysis of the nasal and the paranasal cavities. J Acoust Soc Am. 1994; 96(4):2088-100.

30. Sundberg J, Birch P, Gumoes B, Stavad H, Prytz $S$, Karle A. Experimental findings on the nasal tract resonator in singing. J Voice. 2007; 21(2):127-37.

31. Costa HO, Andrada e Silva MA. Voz cantada: evolução, avaliação e terapia fonoaudiológica. São Paulo: Lovise; 1998. 181p.

32. Farghaly SM, Andrade CRF. Programa de treinamento vocal para locutores de rádio. Rev Soc Bras Fonoaudiol. 2008; 13(4):316-24.

33. Soares EB, Brito CMCP. Perfil vocal do guia de turismo. Rev. CEFAC. 2006; 8(4):501-8. dx.doi.org/ S1516-18462006000400011

34. Alves ASPL. O uso profissional da voz e o medo de falar em público [monografia]. Recife (PE): Universidade Federal de Pernambuco; 2007. 